Pacific

Journal of

Mathematics

RULED MINIMAL SURFACES IN THE THREE-DIMENSIONAL HEISENBERG GROUP

Heayong Shin, Young Wook Kim, Sung-Eun KoH, Hyung Yong LeE AND SEONG-DeOg YANG 


\title{
RULED MINIMAL SURFACES IN THE THREE-DIMENSIONAL HEISENBERG GROUP
}

\author{
Heayong Shin, Young Wook Kim, Sung-Eun Koh, \\ Hyung Yong LeE AND SEONG-Deog YANG
}

To the memory of Professor Seok Woo Kim

\begin{abstract}
It is shown that parts of planes, helicoids and hyperbolic paraboloids are the only minimal surfaces ruled by geodesics in the three-dimensional Riemannian Heisenberg group. It is also shown that they are the only surfaces in the three-dimensional Heisenberg group whose mean curvature is zero with respect to both the standard Riemannian metric and the standard Lorentzian metric.
\end{abstract}

\section{Introduction}

The three-dimensional Heisenberg group $\mathbb{H}_{3}$ is the two-step nilpotent Lie group $\left(\mathbb{R}^{3}, \star\right)$ where

$$
(x, y, z) \star\left(x^{\prime}, y^{\prime}, z^{\prime}\right):=\left(x+x^{\prime}, y+y^{\prime}, z+z^{\prime}+\frac{1}{2}\left(x y^{\prime}-x^{\prime} y\right)\right) .
$$

It is in general identified with a subgroup of $G L_{3}(\mathbb{R})$ by

$$
(x, y, z) \leftrightarrow\left[\begin{array}{ccc}
1 & x & z+\frac{1}{2} x y \\
0 & 1 & y \\
0 & 0 & 1
\end{array}\right] .
$$

We consider in this paper two left-invariant metrics on $\mathbb{H}_{3}$ : one is Riemannian and the other Lorentzian. Let us denote by $\mathrm{Nil}^{3}$ the 3-dimensional Heisenberg group $\mathbb{H}_{3}$ endowed with the left-invariant Riemannian metric

$$
g=d x^{2}+d y^{2}+\left(d z+\frac{1}{2}(y d x-x d y)\right)^{2}
$$

Kim was supported by NRF 2009-0086794, Koh was supported by NRF 2009-0086794 and NRF 2011-0001565 and Yang was supported by NRF 2012R1A1A2042530.

MSC2010: 53A35.

Keywords: Heisenberg group, ruled surface, minimal surface. 
on $\mathbb{R}^{3}$. The Riemannian Heisenberg group $\mathrm{Nil}^{3}$ is a three-dimensional homogeneous manifold with a 4-dimensional isometry group; hence it is the most simple 3manifold apart from the space-forms. Moreover, it is a Riemannian fibration over the Euclidean plane $\mathbb{R}^{2}$, with the projection $(x, y, z) \mapsto(x, y)$.

In the first part of this paper, we give a classification of all ruled minimal surfaces in $\mathrm{Nil}^{3}$. In order to do this, we first show in Lemma 2.1 that if a ruled surface is minimal and if a ruling geodesic is not tangent to the fiber, then the ruled surface should be horizontally ruled. That is, its ruling geodesics are orthogonal to the fibers. In fact, it was one of the key observations in classifying the ruled minimal surfaces in $\mathbb{S}^{2} \times \mathbb{R}$ or in $\mathbb{H}^{2} \times \mathbb{R}$ in our previous paper [Kim et al. 2009a]. It turns out that this fact simplifies the nonlinear partial differential equations describing ruled minimal surfaces. Then we show in Theorem 2.3 that any ruled minimal surface in $\mathrm{Nil}^{3}$ is, up to isometries, a part of the horizontal plane $z=0$, the vertical plane $y=0$, a helicoid $\tan (\lambda z)=y / x, \lambda \neq 0$ or a hyperbolic paraboloid $z=-x y / 2$; see page 480 for the definition of planes. Moreover, we show on pages 488-489 that all of them can be regarded as helicoids or the limits of sequences of helicoids in the Hausdorff sense.

In fact, it was shown in [Bekkar and Sari 1992] that, up to isometries, parts of planes, the helicoids and the hyperbolic paraboloids are the only minimal surfaces in $\mathrm{Nil}^{3}$ ruled by straight lines that are geodesics. According to Lemma 2.1, any ruling geodesic of a ruled minimal surface is either parallel or orthogonal to the fibers. We then note in Proposition 2.4 that geodesics parallel or orthogonal to the fibers everywhere are straight lines (in the Euclidean sense), and thereby show that "straight line" condition may be deleted in the aforementioned claim. For the properties of the Gauss map and representation formulae of the minimal surfaces in $\mathrm{Nil}^{3}$, see for example [Bekkar et al. 2007; Daniel 2011; Inoguchi 2005; 2008; Mercuri et al. 2006; Sanini 1997].

In the second part, we consider the natural left-invariant Lorentzian metric

$$
g_{L}=d x^{2}+d y^{2}-\left(d z+\frac{1}{2}(y d x-x d y)\right)^{2}
$$

on $\mathbb{H}_{3}$. (Lorentzian metrics on $\mathbb{H}_{3}$ are discussed in [Rahmani 1992; Rahmani and Rahmani 2006].) Then we consider surfaces in $\mathbb{H}_{3}$ whose mean curvature is zero with respect to both metrics $g$ and $g_{L}$ and show in Theorem 3.2 that they must be one of the above mentioned surfaces, that is, a part of planes, helicoids or hyperbolic paraboloids. It can be considered as a generalization of the fact that the helicoids are the only surfaces except the planes in $\mathbb{R}^{3}$ whose mean curvature is zero with respect to both the standard Riemannian metric and the standard Lorentzian metric [Kobayashi 1983] and the fact that the helicoids (surfaces invariant under the screw motion) are the only surfaces except the trivial ones in $\mathbb{S}^{2} \times \mathbb{R}$ or $\mathbb{M}^{2} \times \mathbb{R}$ whose mean curvature is zero with respect to both the standard Riemannian metric and the 
standard Lorentzian metric [Kim et al. 2009a]. For this we derive the equation for the mean curvature of a graph in $\mathbb{H}_{3}$ to be zero with respect to the Lorentzian metric $g_{L}$ and compare it with the minimal surface equation. The idea of considering these two equations at the same time is not new - see [Albujer and Alías 2009; Alías and Palmer 2001; Kobayashi 1983]; also, the "dualities" between minimal surfaces in $\mathrm{Nil}^{3}$ and maximal surfaces in the Lorentzian $\mathrm{Nil}^{3}$ were studied in [Lee 2011].

\section{Ruled minimal surfaces in $\mathrm{Nil}^{3}$}

We first state several facts on the geometry of $\mathrm{Nil}^{3}$, necessary for the proof of the main result in this section. For their proofs, one may refer, for example, to [Inoguchi et al. 1999].

A frame field. It can be easily seen that

$$
\boldsymbol{e}_{1}=\frac{\partial}{\partial x}-\frac{y}{2} \frac{\partial}{\partial z}, \quad \boldsymbol{e}_{2}=\frac{\partial}{\partial y}+\frac{x}{2} \frac{\partial}{\partial z}, \quad \boldsymbol{e}_{3}=\frac{\partial}{\partial z}
$$

is a left-invariant orthonormal frame field on $\mathrm{Nil}^{3}$ and in particular, $\boldsymbol{e}_{3}$ is tangent to the fibers. Letting $\nabla$ be the Levi-Civita connection on $\mathrm{Nil}^{3}$, we have for this frame field

$$
\begin{gathered}
\nabla_{\boldsymbol{e}_{i}} \boldsymbol{e}_{i}=0, \quad i=1,2,3, \\
\nabla_{\boldsymbol{e}_{1}} \boldsymbol{e}_{2}=-\nabla_{\boldsymbol{e}_{2}} \boldsymbol{e}_{1}=\frac{1}{2} \boldsymbol{e}_{3}, \quad \nabla_{\boldsymbol{e}_{1}} \boldsymbol{e}_{3}=\nabla_{\boldsymbol{e}_{3}} \boldsymbol{e}_{1}=-\frac{1}{2} \boldsymbol{e}_{2}, \quad \nabla_{\boldsymbol{e}_{2}} \boldsymbol{e}_{3}=\nabla_{\boldsymbol{e}_{3}} \boldsymbol{e}_{2}=\frac{1}{2} \boldsymbol{e}_{1} .
\end{gathered}
$$

Isometries. The isometry group of $\mathrm{Nil}^{3}$ has two connected components: an isometry either preserves the orientation of both the fibers and the base of the fibration, or reverses both orientations. The identity component of the isometry group of $\mathrm{Nil}^{3}$ is isomorphic to $\mathrm{SO}(2) \ltimes \mathbb{R}^{3}$ whose action is given by

$$
\begin{aligned}
\left(\left[\begin{array}{rr}
\cos \theta & -\sin \theta \\
\sin \theta & \cos \theta
\end{array}\right],\left[\begin{array}{l}
a \\
b \\
c
\end{array}\right]\right) \cdot\left[\begin{array}{l}
x \\
y \\
z
\end{array}\right] \\
=\left[\begin{array}{ccc}
\cos \theta & -\sin \theta & 0 \\
\sin \theta & \cos \theta & 0 \\
\frac{1}{2}(a \sin \theta-b \cos \theta) & \frac{1}{2}(a \cos \theta+b \sin \theta) & 1
\end{array}\right]\left[\begin{array}{c}
c x \\
y \\
z
\end{array}\right]+\left[\begin{array}{l}
a \\
b \\
c
\end{array}\right],
\end{aligned}
$$

which shows that $\mathrm{Nil}^{3}$ is a homogeneous space. In fact, one can see that, for any point $p \in \mathbb{H}_{3}$ and a unit tangent vector $\boldsymbol{v}$ orthogonal to $\boldsymbol{e}_{3}(p)$, there exists a unique isometry $\varphi$ such that $\varphi(p)=\mathbf{0}, d \varphi(\boldsymbol{v})=\boldsymbol{e}_{1}(\mathbf{0})$ and $d \varphi\left(\boldsymbol{e}_{3}(p)\right)=\boldsymbol{e}_{3}(\mathbf{0})$. Note also that the translations along the $z$-axis (in the Euclidean sense) are isometries belonging to the identity component. 
Euclidean planes. A Euclidean plane or simply a plane is a set of points $(x, y, z) \in$ $\mathbb{H}_{3}$ satisfying a linear equation $a x+b y+c z+d=0$. It is easy to see that all the planes except the "vertical" planes $a x+b y+d=0$ are congruent. In fact, every nonvertical plane $a x+b y+z+d=0$ is congruent to the "horizontal" plane $z=0$ under the left translation by $(-2 b, 2 a,-d),(x, y, z) \mapsto(-2 b, 2 a,-d) \star(x, y, z)$, that is,

$$
\left[\begin{array}{l}
x \\
y \\
z
\end{array}\right] \mapsto\left[\begin{array}{ccc}
1 & 0 & 1 \\
0 & 1 & 0 \\
-a & -b & 1
\end{array}\right]\left[\begin{array}{c}
c x \\
y \\
z
\end{array}\right]+\left[\begin{array}{c}
r-2 b \\
2 a \\
-d
\end{array}\right] .
$$

Moreover, a vertical plane is not congruent to a nonvertical plane since every isometric image of a fiber is a fiber. In fact, one can check that a vertical plane is not isometric to a nonvertical plane by computing their curvatures.

A parametrization of ruled surfaces. Let $\Sigma$ be a ruled surface in $\mathrm{Nil}^{3}$ and let $p \in \Sigma$ be a point at which $T_{p} \Sigma$ is transversal to the fiber. Assume, furthermore, that the direction of the ruling geodesic at $p$ is not perpendicular to the fibers. Then, in a neighborhood of $p$, we can take a tangent vector field $V$ to $\Sigma$ in the direction of the ruling (everywhere on the neighborhood) as

$$
V=\eta\left(\cos \theta \boldsymbol{e}_{1}-\sin \theta \boldsymbol{e}_{2}\right)+\boldsymbol{e}_{3}
$$

for some functions $\eta$ and $\theta$ on $\Sigma$. Since $T_{p} \Sigma$ is transversal to the fiber, the unit normal vector field $\boldsymbol{n}$ of $\Sigma$ is not perpendicular to $\boldsymbol{e}_{3}:\left\langle\boldsymbol{n}, \boldsymbol{e}_{3}\right\rangle \neq 0$. Then

$$
W=\sin \theta \boldsymbol{e}_{1}+\cos \theta \boldsymbol{e}_{2}-\frac{\left\langle\boldsymbol{n}, \sin \theta \boldsymbol{e}_{1}+\cos \theta \boldsymbol{e}_{2}\right\rangle}{\left\langle\boldsymbol{n}, \boldsymbol{e}_{3}\right\rangle} \boldsymbol{e}_{3}
$$

gives another tangent vector field on $\Sigma$ which is transversal to $V$. Now we take a parametrization $X(s, t)$ of $\Sigma$ in the neighborhood of $p$ such that $X(s, 0)$ is the integral curve of $W$ with $X(0,0)=p$ and such that $t$ parameter curves are the ruling geodesics with $X_{t}(s, 0)=V(X(s, 0))$. Then $X(s, t)$ is a parametrization of the ruled surface $\Sigma$ in the neighborhood of $p$ satisfying

$$
\begin{aligned}
X_{s}(s, 0) & =\sin \alpha(s) \boldsymbol{e}_{1}+\cos \alpha(s) \boldsymbol{e}_{2}+g(s) \boldsymbol{e}_{3}, \\
X_{t}(s, 0) & =h(s)\left(\cos \alpha(s) \boldsymbol{e}_{1}-\sin \alpha(s) \boldsymbol{e}_{2}\right)+\boldsymbol{e}_{3}, \\
\nabla_{X_{t}} X_{t} & =0,
\end{aligned}
$$

for some smooth functions $h(s), \alpha(s)$ and $g(s)$.

For the parametrization $X$ satisfying the condition (1), we are to compute the functions $X_{s i}$ and $X_{t i}$ defined by

$$
\begin{aligned}
& X_{s}(s, t)=X_{s 1}(s, t) \boldsymbol{e}_{1}+X_{s 2}(s, t) \boldsymbol{e}_{2}+X_{s 3}(s, t) \boldsymbol{e}_{3}, \\
& X_{t}(s, t)=X_{t 1}(s, t) \boldsymbol{e}_{1}+X_{t 2}(s, t) \boldsymbol{e}_{2}+X_{t 3}(s, t) \boldsymbol{e}_{3} .
\end{aligned}
$$


Now, since $t$ parameter curves are geodesics, we have

$$
\begin{aligned}
\nabla_{X_{t}} X_{t} & =\sum_{i} \frac{\partial X_{t i}}{\partial t} \boldsymbol{e}_{i}+\sum_{i, j} X_{t i} X_{t j} \nabla_{\boldsymbol{e}_{i}} \boldsymbol{e}_{j} \\
& =\left(\frac{\partial X_{t 1}}{\partial t}+X_{t 2} X_{t 3}\right) \boldsymbol{e}_{1}+\left(\frac{\partial X_{t 2}}{\partial t}-X_{t 1} X_{t 3}\right) \boldsymbol{e}_{2}+\frac{\partial X_{t 3}}{\partial t} \boldsymbol{e}_{3}=0 .
\end{aligned}
$$

By solving the system of equations

$$
\frac{\partial X_{t 1}}{\partial t}+X_{t 2} X_{t 3}=0, \quad \frac{\partial X_{t 2}}{\partial t}-X_{t 1} X_{t 3}=0, \quad \frac{\partial X_{t 3}}{\partial t}=0
$$

with the initial condition

$$
X_{t 1}(s, 0)=h(s) \cos \alpha(s), \quad X_{t 2}(s, 0)=-h(s) \sin \alpha(s), \quad X_{t 3}(s, 0)=1,
$$

we have

$$
X_{t 1}(s, t)=h(s) \cos (t-\alpha(s)), \quad X_{t 2}(s, t)=h(s) \sin (t-\alpha(s)), \quad X_{t 3}(s, t)=1 .
$$

On the other hand, since the Levi-Civita connection $\nabla$ is torsion-free, one has

$$
\nabla_{X_{t}} X_{s}=\nabla_{X_{s}} X_{t}
$$

Hence we have

$$
\begin{aligned}
&\left(\frac{\partial X_{s 1}}{\partial t}+\frac{1}{2}\left(X_{t 2} X_{s 3}+X_{t 3} X_{s 2}\right)\right) \boldsymbol{e}_{1}+\left(\frac{\partial X_{s 2}}{\partial t}\right.\left.-\frac{1}{2}\left(X_{t 1} X_{s 3}+X_{t 3} X_{s 1}\right)\right) \boldsymbol{e}_{2} \\
&+\left(\frac{\partial X_{s 3}}{\partial t}+\frac{1}{2}\left(X_{t 1} X_{s 2}-X_{t 2} X_{s 1}\right)\right) \boldsymbol{e}_{3} \\
&=\left(\frac{\partial X_{t 1}}{\partial s}+\frac{1}{2}\left(X_{s 2} X_{t 3}+X_{s 3} X_{t 2}\right)\right) \boldsymbol{e}_{1}+\left(\frac{\partial X_{t 2}}{\partial s}-\frac{1}{2}\left(X_{s 1} X_{t 3}+X_{s 3} X_{t 1}\right)\right) \boldsymbol{e}_{2} \\
&+\left(\frac{\partial X_{t 3}}{\partial s}+\frac{1}{2}\left(X_{s 1} X_{t 2}-X_{s 2} X_{t 1}\right)\right) \boldsymbol{e}_{3},
\end{aligned}
$$

and $X_{s i}$ satisfies the equations

$$
\begin{aligned}
\frac{\partial X_{s 1}}{\partial t} & =\frac{\partial X_{t 1}}{\partial s}=h^{\prime}(s) \cos (t-\alpha(s))+h(s) \alpha^{\prime}(s) \sin (t-\alpha(s)) \\
\frac{\partial X_{s 2}}{\partial t} & =\frac{\partial X_{t 2}}{\partial s}=h^{\prime}(s) \sin (t-\alpha(s))-h(s) \alpha^{\prime}(s) \cos (t-\alpha(s)) \\
\frac{\partial X_{s 3}}{\partial t} & =\frac{\partial X_{t 3}}{\partial s}+\left(X_{s 1} X_{t 2}-X_{s 2} X_{t 1}\right) \\
& =h(s) \sin (t-\alpha(s)) X_{s 1}-h(s) \cos (t-\alpha(s)) X_{s 2}
\end{aligned}
$$

with the initial condition

$$
X_{s 1}(s, 0)=\sin \alpha(s), \quad X_{s 2}(s, 0)=\cos \alpha(s), \quad X_{s 3}(s, 0)=g(s) .
$$


By solving these equations, we get

$$
\begin{gathered}
X_{s 1}(s, t)=\sin \alpha(s)+h^{\prime}(s) \sin (t-\alpha(s))+h^{\prime}(s) \sin \alpha(s) \\
-h(s) \alpha^{\prime}(s) \cos (t-\alpha(s))+h(s) \alpha^{\prime}(s) \cos \alpha(s), \\
\begin{array}{r}
X_{s 2}(s, t)=\cos \alpha(s)-h^{\prime}(s) \cos (t-\alpha(s))+h^{\prime}(s) \cos \alpha(s) \\
-h(s) \alpha^{\prime}(s) \sin (t-\alpha(s))-h(s) \alpha^{\prime}(s) \sin \alpha(s), \\
X_{s 3}(s, t)=g(s)-h(s) \sin t+t h(s) h^{\prime}(s)-h(s) h^{\prime}(s) \sin t \\
+h(s)^{2} \alpha^{\prime}(s)-h(s)^{2} \alpha^{\prime}(s) \cos t .
\end{array}
\end{gathered}
$$

The second derivatives of $\boldsymbol{X}$. We will compute the derivatives

$$
\nabla_{X_{t}} X_{t}, \quad \nabla_{X_{s}} X_{t}=\nabla_{X_{t}} X_{s} \quad \text { and } \quad \nabla_{X_{s}} X_{s}
$$

For notational simplicity, let us set

$$
\begin{aligned}
& X_{t ; t}:=\nabla_{X_{t}} X_{t}=X_{t t 1} \boldsymbol{e}_{1}+X_{t t 2} \boldsymbol{e}_{2}+X_{t t 3} \boldsymbol{e}_{3}, \\
& X_{s ; t}:=\nabla_{X_{t}} X_{s}=X_{s t 1} \boldsymbol{e}_{1}+X_{s t 2} \boldsymbol{e}_{2}+X_{s t 3} \boldsymbol{e}_{3}, \\
& X_{s ; s}:=\nabla_{X_{s}} X_{s}=X_{s s 1} \boldsymbol{e}_{1}+X_{s s 2} \boldsymbol{e}_{2}+X_{s s 3} \boldsymbol{e}_{3} .
\end{aligned}
$$

Since $t$ parameter curves are geodesics, we have $X_{t ; t}=0$, that is,

$$
X_{t t 1}=X_{t t 2}=X_{t t 3}=0 \text {. }
$$

From the equalities

$$
\begin{aligned}
X_{s ; t}= & X_{t ; s}=\left(\frac{\partial X_{s 1}}{\partial t}+\frac{1}{2}\left(X_{t 2} X_{s 3}+X_{t 3} X_{s 2}\right)\right) \boldsymbol{e}_{1} \\
& +\left(\frac{\partial X_{s 2}}{\partial t}-\frac{1}{2}\left(X_{t 1} X_{s 3}+X_{t 3} X_{s 1}\right)\right) \boldsymbol{e}_{2}+\left(\frac{\partial X_{s 3}}{\partial t}+\frac{1}{2}\left(X_{t 1} X_{s 2}-X_{t 2} X_{s 1}\right)\right) \boldsymbol{e}_{3}, \\
X_{s ; s}= & \left(\frac{\partial X_{s 1}}{\partial s}+X_{s 2} X_{s 3}\right) \boldsymbol{e}_{1}+\left(\frac{\partial X_{s 2}}{\partial s}-X_{s 1} X_{s 3}\right) \boldsymbol{e}_{2}+\frac{\partial X_{s 3}}{\partial s} \boldsymbol{e}_{3}
\end{aligned}
$$

we have

$$
\begin{array}{r}
X_{s t 1}=\frac{1}{2}\left[\cos \alpha(s)+h^{\prime}(s) \cos (t-\alpha(s))+h^{\prime}(s) \cos \alpha(s)\right. \\
+h(s) \alpha^{\prime}(s) \sin (t-\alpha(s))-h(s) \alpha^{\prime}(s) \sin \alpha(s)+h(s) \sin (t-\alpha(s))(g(s) \\
\left.\left.+h(s)\left(-\sin t+h^{\prime}(s)(t-\sin t)+2 h(s) \alpha^{\prime}(s) \sin ^{2} t / 2\right)\right)\right], \\
X_{s t 2}=\frac{1}{2}\left[-\sin \alpha(s)+h^{\prime}(s) \sin (t-\alpha(s))-h^{\prime}(s) \sin \alpha(s)-h(s) \alpha^{\prime}(s) \cos (t-\alpha(s))\right. \\
-h(s) \alpha^{\prime}(s) \cos \alpha(s)+h(s) \cos (t-\alpha(s))(-g(s) \\
\left.\left.+h(s)\left(\sin t-h^{\prime}(s)(t-\sin t)-2 h(s) \alpha^{\prime}(s) \sin ^{2} t / 2\right)\right)\right],
\end{array}
$$

$X_{s t 3}=\frac{1}{2} h(s)\left[-\cos t-h^{\prime}(s)(\cos t-1)+h(s) \alpha^{\prime}(s) \sin t\right]$, 


$$
\begin{aligned}
X_{s s 1}=\alpha^{\prime}(s) & \cos \alpha(s)-2 h^{\prime}(s) \alpha^{\prime}(s) \cos (t-\alpha(s))+2 h^{\prime}(s) \alpha^{\prime}(s) \cos \alpha(s) \\
- & h(s) \alpha^{\prime}(s)^{2} \sin (t-\alpha(s))-h(s) \alpha^{\prime}(s)^{2} \sin \alpha(s) \\
+ & \left(-\cos \alpha(s)+\cos (t-\alpha(s))-h^{\prime}(s) \cos \alpha(s)\right. \\
& \left.+h(s) \sin (t-\alpha(s))+\alpha^{\prime}(s) \sin \alpha(s)\right) \\
& \times\left(-g(s)+h(s)\left(\sin t+h^{\prime}(s)(\sin t-t)-2 h(s) \alpha^{\prime}(s) \sin ^{2} t / 2\right)\right) \\
+ & h^{\prime \prime}(s) \sin (t-\alpha(s))+h^{\prime \prime}(s) \sin \alpha(s) \\
- & h(s) \alpha^{\prime \prime}(s) \cos (t-\alpha(s))+h(s) \alpha^{\prime \prime}(s) \cos \alpha(s), \\
X_{s s 2}=-\alpha^{\prime}(s) & \sin \alpha(s)-2 h^{\prime}(s) \alpha^{\prime}(s) \sin (t-\alpha(s))-2 h^{\prime}(s) \alpha^{\prime}(s) \sin \alpha(s) \\
+ & h(s) \alpha^{\prime}(s)^{2} \cos (t-\alpha(s))-h(s) \alpha^{\prime}(s)^{2} \cos \alpha(s) \\
+ & \left(\sin \alpha(s)+h^{\prime}(s)(\sin (t-\alpha(s))+\sin \alpha(s))\right. \\
& \left.+2 h(s) \alpha^{\prime}(s) \sin t / 2 \sin (t / 2-\alpha(s))\right) \\
& \times\left(-g(s)+h(s)\left(\sin t+h^{\prime}(s)(\sin t-t)-2 h(s) \alpha^{\prime}(s) \sin ^{2} t / 2\right)\right) \\
- & h^{\prime \prime}(s) \cos (t-\alpha(s))+h^{\prime \prime}(s) \cos \alpha(s) \\
- & h(s) \alpha^{\prime \prime}(s) \sin (t-\alpha(s))-h(s) \alpha^{\prime \prime}(s) \sin \alpha(s),
\end{aligned}
$$

$$
\begin{aligned}
X_{s s 3}=g^{\prime}(s)+h^{\prime}(s)^{2}(t-\sin t)- & h^{\prime}(s)\left(\sin t-4 h(s) \alpha^{\prime}(s) \sin ^{2} t / 2\right) \\
& +h(s)\left(h^{\prime \prime}(s)(t-\sin t)-h(s) \alpha^{\prime \prime}(s)(\cos t-1)\right) .
\end{aligned}
$$

Mean curvature. We give a condition for the ruled surface $\Sigma$ to be minimal in terms of the parametrization $X$. Let $E, F, G$ and $l, m, n$, respectively, be the coefficients of the first and second fundamental forms of the surface $\Sigma$ whose parametrization satisfies (1). Then the mean curvature of $\Sigma$ in a neighborhood of $p$ is given by

$$
H=\frac{1}{2} \frac{G l-2 F m+E n}{E G-F^{2}}=\frac{1}{2} \frac{\left\langle X_{t}, X_{t}\right\rangle\left\langle X_{s ; s}, X_{s} \times X_{t}\right\rangle-2\left\langle X_{s}, X_{t}\right\rangle\left\langle X_{s ; t}, X_{s} \times X_{t}\right\rangle}{\left\|X_{s} \times X_{t}\right\|^{3}} .
$$

Since

$X_{s} \times X_{t}=\left(X_{s 2} X_{t 3}-X_{s 3} X_{t 2}\right) \boldsymbol{e}_{1}+\left(X_{s 3} X_{t 1}-X_{s 1} X_{t 3}\right) \boldsymbol{e}_{2}+\left(X_{s 1} X_{t 2}-X_{s 2} X_{t 1}\right) \boldsymbol{e}_{3}$, $X$ is a parametrization of a minimal surface if and only if

$$
\begin{aligned}
\tilde{H}:= & \left\langle X_{t}, X_{t}\right\rangle\left\langle X_{s ; s}, X_{s} \times X_{t}\right\rangle-2\left\langle X_{s}, X_{t}\right\rangle\left\langle X_{s ; t}, X_{s} \times X_{t}\right\rangle \\
= & \left(\sum_{i} X_{t i}^{2}\right)\left(\left(X_{s 2} X_{t 3}-X_{s 3} X_{t 2}\right) X_{s s 1}+\left(X_{s 3} X_{t 1}-X_{s 1} X_{t 3}\right) X_{s s 2}\right. \\
& \left.\quad+\left(X_{s 1} X_{t 2}-X_{s 2} X_{t 1}\right) X_{s s 3}\right) \\
& -2\left(\sum_{i} X_{s i} X_{t i}\right)\left(\left(X_{s 2} X_{t 3}-X_{s 3} X_{t 2}\right) X_{s t 1}\right. \\
& \left.+\left(X_{s 3} X_{t 1}-X_{s 1} X_{t 3}\right) X_{s t 2}+\left(X_{s 1} X_{t 2}-X_{s 2} X_{t 1}\right) X_{s t 3}\right)
\end{aligned}
$$$$
=0 \text {. }
$$ 
Ruled minimal surfaces in $\mathrm{Nil}^{3}$. Now we will find all ruled minimal surfaces in $\mathrm{Nil}^{3}$.

Lemma 2.1. If the surface whose parametrization $X$ satisfies (1) is minimal, then $h(s)=0$ for all $s$.

Proof. Considering the parametrizations $\tilde{X}(s, t):=X\left(s-s_{0}, t\right)$ if necessary, we need only to prove $h(0)=0$. By rotating the surface in $\mathrm{Nil}^{3}$ if necessary, we may assume that $\alpha(0)=0$. Since we have explicit formulae for all $X_{s}, X_{t}, X_{s ; s}$, $X_{s ; t}, X_{t ; t}$, we can compute $\tilde{H}$ directly. In particular, since $X$ is minimal, we have $\tilde{H}(0, t)=0$ for all $t$. Since $\alpha(0)=0, \tilde{H}(0, t)$ becomes

$$
\begin{aligned}
\tilde{H}(0, t)=A_{0} & +A_{1} t+A_{2} t^{2}+A_{3} t^{3} \\
+ & B_{0} \cos t+B_{1} t \cos t+B_{2} t^{2} \cos t+B_{3} \cos 2 t+B_{4} t \cos 2 t+B_{5} \cos 3 t \\
& +C_{0} \sin t+C_{1} t \sin t+C_{2} t^{2} \sin t+C_{3} \sin 2 t+C_{4} t \sin 2 t+C_{5} \sin 3 t
\end{aligned}
$$

where the constants $A_{i}, B_{i}, C_{i}$ are functions of $h(0), h^{\prime}(0), h^{\prime \prime}(0), \alpha^{\prime}(0), \alpha^{\prime \prime}(0)$ and $g(0), g^{\prime}(0)$. In the following computation, we are to use only the following terms:

$$
\begin{aligned}
& A_{3}=h(0)^{5} h^{\prime}(0)^{3}, \\
& B_{1}=-3 h(0) h^{\prime}(0)^{2}-h(0)^{3} h^{\prime}(0)^{2}-3 h(0) h^{\prime}(0)^{3}-h(0)^{3} h^{\prime}(0)^{3} \\
& \quad-2 h(0)^{3} g(0) h^{\prime}(0) \alpha^{\prime}(0)-6 g(0) h(0)^{5} h^{\prime}(0) \alpha^{\prime}(0)-3 h(0)^{3} h^{\prime}(0) \alpha^{\prime}(0)^{2} \\
& -9 h^{\prime}(0) h(0)^{5} \alpha^{\prime}(0)^{2}-6 h(0)^{7} h^{\prime}(0) \alpha^{\prime}(0)^{2}-h(0)^{4} h^{\prime \prime}(0)-h(0)^{2} h^{\prime \prime}(0), \\
& B_{5}=\frac{1}{4}\left(3 h(0)^{4} \alpha^{\prime}(0)+3 h(0)^{6} \alpha^{\prime}(0)+6 h(0)^{4} h^{\prime}(0) \alpha^{\prime}(0)+6 h(0)^{6} h^{\prime}(0) \alpha^{\prime}(0)\right. \\
& \left.+3 h(0)^{4} h^{\prime}(0)^{2} \alpha^{\prime}(0)+3 h^{\prime}(0)^{2} \alpha^{\prime}(0) h(0)^{6}-h(0)^{6} \alpha^{\prime}(0)^{3}-h(0)^{8} \alpha^{\prime}(0)^{3}\right), \\
& C_{5}=\frac{1}{4}\left(h(0)^{3}+h(0)^{5}+3 h(0)^{3} h^{\prime}(0)+3 h(0)^{5} h^{\prime}(0)+3 h(0)^{3} h^{\prime}(0)^{2}\right. \\
& +3 h(0)^{5} h^{\prime}(0)^{2}+h(0)^{3} h^{\prime}(0)^{3}+h(0)^{5} h^{\prime}(0)^{3}-3 h(0)^{5} \alpha^{\prime}(0)^{2} \\
& \left.-3 h(0)^{7} \alpha^{\prime}(0)^{2}-3 h(0)^{5} h^{\prime}(0) \alpha^{\prime}(0)^{2}-3 h^{\prime}(0) h(0)^{7} \alpha^{\prime}(0)^{2}\right) .
\end{aligned}
$$

Since $\tilde{H}(0, t)=0$ for all $t$ and since the above expression is a linear combination of linearly independent functions of $t$, all of $A_{i}, B_{i}, C_{i}$ must be 0 . Since $A_{3}=$ $h(0)^{5} h^{\prime}(0)^{3}=0$, we have either $h(0)=0$ or $h^{\prime}(0)=0$. Now suppose $h(0) \neq 0$. Then $h^{\prime}(0)=0$ and $B_{1}$ becomes

$$
B_{1}=-h^{\prime \prime}(0) h(0)^{4}-h^{\prime \prime}(0) h(0)^{2}=-h^{\prime \prime}(0) h(0)^{2}\left(h(0)^{2}+1\right)=0 .
$$

Hence we have $h^{\prime \prime}(0)=0$ and in addition

$$
\begin{aligned}
& 4 B_{5}=-\alpha^{\prime}(0)^{3} h(0)^{8}-\alpha^{\prime}(0)^{3} h(0)^{6}+3 \alpha^{\prime}(0) h(0)^{6}+3 \alpha^{\prime}(0) h(0)^{4}=0, \\
& 4 C_{5}=-3 \alpha^{\prime}(0)^{2} h(0)^{7}-3 \alpha^{\prime}(0)^{2} h(0)^{5}+h(0)^{5}+h(0)^{3}=0 .
\end{aligned}
$$


Then, since $3 B_{5}-h(0) \alpha^{\prime}(0) C_{5}=2 \alpha^{\prime}(0) h(0)^{4}\left(h(0)^{2}+1\right)=0$, we have $\alpha^{\prime}(0)=0$ and $C_{5}$ becomes $4 C_{5}=h(0)^{3}\left(h(0)^{2}+1\right)=0$. This contradicts the assumption $h(0) \neq 0$. Hence we must have $h(0)=0$ if $X$ is a parametrization of a minimal surface.

If $p$ is a point in a ruled surface $\Sigma$ at which $T_{p} \Sigma$ is transversal to the fiber and the direction of the ruling is not perpendicular to the fibers, then $\Sigma$ has the parametrization of the type given in (1) in a neighborhood of $p$. If, in addition, $\Sigma$ is minimal then the above lemma implies that the direction of the ruling at $p$ is parallel to the fibers. This contradicts the fact that $T_{p} \Sigma$ is transversal to the fibers. Therefore we can conclude that in a ruled minimal surface $\Sigma$ the directions of the rulings are horizontal, that is, perpendicular to the fibers wherever $T_{p} \Sigma$ is transversal to the fibers.

Now we consider the minimal surfaces which are ruled by horizontal geodesics.

Lemma 2.2. If $\Sigma$ is a minimal surface in $\mathrm{Nil}^{3}$ ruled by geodesics perpendicular to the fibers, then up to the isometries in $\mathrm{Nil}^{3}, \Sigma$ is a part of the horizontal plane $z=0$, the vertical plane $y=0$, a helicoid $\tan (\lambda z)=y / x, \lambda \neq 0$ or a hyperbolic paraboloid $z=-x y / 2$.

Proof. One can see that the surface $\Sigma$ has a local parametrization $Y(s, t)$ satisfying

$$
\begin{aligned}
Y_{s}(s, 0) & =\cos \beta(s)\left(-\sin \alpha(s) \boldsymbol{e}_{1}+\cos \alpha(s) \boldsymbol{e}_{2}\right)+\sin \beta(s) \boldsymbol{e}_{3}, \\
Y_{t}(s, 0) & =\cos \alpha(s) \boldsymbol{e}_{1}+\sin \alpha(s) \boldsymbol{e}_{2}, \\
\nabla_{Y_{t}} Y_{t} & =0 .
\end{aligned}
$$

If we set

$$
\begin{gathered}
Y_{s}(s, t)=Y_{s 1}(s, t) \boldsymbol{e}_{1}+Y_{s 2}(s, t) \boldsymbol{e}_{2}+Y_{s 3}(s, t) \boldsymbol{e}_{3}, \\
Y_{t}(s, t)=Y_{t 1}(s, t) \boldsymbol{e}_{1}+Y_{t 2}(s, t) \boldsymbol{e}_{2}+Y_{t 3}(s, t) \boldsymbol{e}_{3},
\end{gathered}
$$

by solving the equation $\nabla_{Y_{t}} Y_{t}=0$ with the initial condition

$$
Y_{t}(s, 0)=\cos \alpha(s) \boldsymbol{e}_{1}+\sin \alpha(s) \boldsymbol{e}_{2},
$$

we have

$$
Y_{t 1}(s, t)=\cos \alpha(s), \quad Y_{t 2}(s, t)=\sin \alpha(s), \quad Y_{t 3}(s, t)=0 .
$$

Moreover, from $\nabla_{Y_{t}} Y_{s}=\nabla_{Y_{s}} Y_{t}$, we can see that $Y_{s i}$ satisfies the equations

$$
\begin{aligned}
& \frac{\partial Y_{s 1}}{\partial t}=\frac{\partial Y_{t 1}}{\partial s}=-\alpha^{\prime}(s) \sin \alpha(s) \\
& \frac{\partial Y_{s 2}}{\partial t}=\frac{\partial Y_{t 2}}{\partial s}=\alpha^{\prime}(s) \cos \alpha(s), \\
& \frac{\partial Y_{s 3}}{\partial t}=\frac{\partial Y_{t 3}}{\partial s}+\left(Y_{s 1} Y_{t 2}-Y_{s 2} Y_{t 1}\right)=\sin \alpha(s) Y_{s 1}-\cos \alpha(s) Y_{s 2}
\end{aligned}
$$


with the initial condition

$Y_{s 1}(s, 0)=-\cos \beta(s) \sin \alpha(s), Y_{s 2}(s, 0)=\cos \beta(s) \cos \alpha(s), Y_{s 3}(s, 0)=\sin \beta(s)$.

By solving this system of equations, we get

$$
\begin{aligned}
& Y_{s 1}(s, t)=-\cos \beta(s) \sin \alpha(s)-t \alpha^{\prime}(s) \sin \alpha(s), \\
& Y_{s 2}(s, t)=\cos \beta(s) \cos \alpha(s)+t \alpha^{\prime}(s) \cos \alpha(s), \\
& Y_{s 3}(s, t)=\sin \beta(s)-t \cos \beta(s)-\frac{1}{2} t^{2} \alpha^{\prime}(s) .
\end{aligned}
$$

By direct computations, we can see that the minimal surface (2) can be written as

$$
\begin{aligned}
\beta^{\prime}(s)+t\left(\alpha^{\prime}(s) \beta^{\prime}(s) \cos \beta(s)-\alpha^{\prime \prime}\right. & (s) \sin \beta(s)) \\
& +\frac{t^{2}}{2}\left(\alpha^{\prime}(s) \beta^{\prime}(s) \sin \beta(s)+\alpha^{\prime \prime}(s) \cos \beta(s)\right)=0 .
\end{aligned}
$$

Therefore we have $\beta^{\prime}(s)=0$ and $\alpha^{\prime \prime}(s)=0$, that is, $\beta(s)=b$ and $\alpha(s)=a s+c$ for some constants $a, b, c$.

When $a \neq 0$, relocating the surface $\Sigma$ by an isometry in $\mathrm{Nil}^{3}$, we may assume that

$$
\alpha(s)=a s \quad \text { and } \quad Y(0,0)=\left(\frac{\cos b}{a}, 0,0\right) .
$$

Then, since

$$
\boldsymbol{e}_{1}=\frac{\partial}{\partial x}-\frac{y}{2} \frac{\partial}{\partial z}, \quad \boldsymbol{e}_{2}=\frac{\partial}{\partial y}+\frac{x}{2} \frac{\partial}{\partial z}, \quad \boldsymbol{e}_{3}=\frac{\partial}{\partial z},
$$

we have

$$
\begin{aligned}
Y_{s}(s, 0)= & -\cos b \sin (a s) \boldsymbol{e}_{1}+\cos b \cos (a s) \boldsymbol{e}_{2}+\sin b \boldsymbol{e}_{3} \\
= & -\cos b \sin (a s) \frac{\partial}{\partial x}+\cos b \cos (a s) \frac{\partial}{\partial y} \\
& \quad+\left(\sin b+\frac{y}{2} \cos b \sin (a s)+\frac{x}{2} \cos b \cos (a s)\right) \frac{\partial}{\partial z}, \\
Y_{t}(s, t)= & \cos (a s) \boldsymbol{e}_{1}+\sin (a s) \boldsymbol{e}_{2} \\
= & \cos (a s) \frac{\partial}{\partial x}+\sin (a s) \frac{\partial}{\partial y}+\left(-\frac{y}{2} \cos (a s)+\frac{x}{2} \sin (a s)\right) \frac{\partial}{\partial z} .
\end{aligned}
$$

Integrating the components of $Y_{s}(s, 0)$ with initial data $Y(0,0)=((\cos b) / a, 0,0)$, we have

$$
Y(s, 0)=\left(\frac{1}{a} \cos b \cos (a s), \frac{1}{a} \cos b \sin (a s), m \frac{s}{4 a}(1+\cos (2 b)+4 a \sin b)\right) .
$$


Then integrating the components of $Y_{t}(s, t)$ with initial data $Y(s, 0)$, we have

$$
\begin{aligned}
Y(s, t)=\left(t \cos (a s)+\frac{1}{a} \cos b \cos (a s),\right. \\
\left.\qquad \sin (a s)+\frac{1}{a} \cos b \sin (a s), \frac{s}{4 a}(1+\cos (2 b)+4 a \sin b)\right) .
\end{aligned}
$$

Noting that

$$
Y(s, t)=\left(t \cos (a s), t \sin (a s), \frac{s}{4 a}(1+\cos (2 b)+4 \sin b)\right),
$$

we can see that $Y$ is a parametrization of either the helicoid

$$
\tan \lambda z=\frac{y}{x} \quad \text { where } \lambda=\frac{4 a^{2}}{1+\cos (2 b)+4 a \sin b}
$$

if $1+\cos (2 b)+4 a \sin b \neq 0$, or the plane $z=0$ if $1+\cos (2 b)+4 a \sin b=0$.

When $a=0$ and $\cos b \neq 0$, we may assume up to isometries that $\alpha(s)=0$ and $Y(0,0)=(-\tan b, 0,0)$. Then

$$
\begin{aligned}
Y_{s}(s, 0) & =\cos b \boldsymbol{e}_{2}+\sin b \boldsymbol{e}_{3}=\cos b \frac{\partial}{\partial y}+\left(\sin b+\frac{x}{2} \cos b\right) \frac{\partial}{\partial z}, \\
Y_{t}(s, t) & =\boldsymbol{e}_{1}=\frac{\partial}{\partial x}-\frac{y}{2} \frac{\partial}{\partial z},
\end{aligned}
$$

and a similar computation as above gives

$$
Y(s, t)=\left(t-\tan b, s \cos b,-\frac{1}{2} s t \cos b+\frac{1}{2} s \sin b\right),
$$

which is a parametrization of the hyperbolic paraboloid $z=-x y / 2$. When $a=0$ and $\cos b=0$, we have

$$
Y_{s}(s, 0)=\boldsymbol{e}_{3}, \quad Y_{t}(s, t)=\boldsymbol{e}_{1}=\frac{\partial}{\partial x}-\frac{y}{2} \frac{\partial}{\partial z}
$$

and $Y(s, t)$ is a parametrization of the $x z$-plane if we set $Y(0,0)=(0,0,0)$.

Theorem 2.3. If $\Sigma$ is a minimal surface in $\mathrm{Nil}^{3}$ ruled by geodesics, then up to the isometries in $\mathrm{Nil}^{3}, \Sigma$ is a part of the horizontal plane $z=0$, the vertical plane $y=0$, a helicoid $\tan (\lambda z)=y / x, \lambda \neq 0$ or a hyperbolic paraboloid $z=-x y / 2$.

Proof. If there is a point $p \in \Sigma$ at which $T_{p} \Sigma$ is transversal to the fibers, then $\Sigma$ is transversal to the fibers in a neighborhood of $p$. Therefore, from the argument following Lemma 2.1, the ruling geodesics through any points in the neighborhood must be horizontal. Then by Lemma 2.2 the neighborhood coincides with a part of the helicoids, the hyperbolic paraboloid or the $x y$-plane up to the isometries in $\mathrm{Nil}^{3}$. Now since the tangent spaces at every point of these surfaces are transversal to fibers, the whole $\Sigma$ must be a part of one of these surfaces. 
On the other hand, if the tangent space $T_{p} \Sigma$ is tangent to the fibers at every point $p \in \Sigma$, then $\boldsymbol{e}_{3}$ is tangent to $\Sigma$. Relocating $\Sigma$ by an isometry of $\mathrm{Nil}^{3}$, we may assume that $(0,0,0) \in \Sigma$ and that $\Sigma$ is tangent to the plane $y=0$ at $(0,0,0)$. So $\Sigma$ is ruled by the fibers and has a ruled parametrization $X(s, t)=(x(s), y(s), t)$ satisfying $x(0)=y(0)=0, y^{\prime}(0)=0$ and $x^{\prime}(0)=1$. The mean curvature of this parametrized surface can be easily computed to be

$$
\frac{x^{\prime \prime}(s) y^{\prime}(s)-x^{\prime}(s) y^{\prime \prime}(s)}{\left(x^{\prime}(s)^{2}+y^{\prime}(s)^{2}\right)^{3 / 2}} .
$$

Solving the equation $x^{\prime \prime}(s) y^{\prime}(s)-x^{\prime}(s) y^{\prime \prime}(s)=0$ with the above initial conditions, we have $y(s)=0$, which implies that $\Sigma$ is a part of the vertical plane $y=0$.

We remark that the mean curvature formula of the cylinder over curves in the $x y$-plane is given in p. 22 of [Inoguchi et al. 2000], and that characterizations of these cylinders in terms of the harmonicity of the tangential Gauss maps are given in [Sanini 1997].

By the above theorem, we know that the ruled minimal surfaces in $\mathrm{Nil}^{3}$ are congruent to the surfaces given in the theorem, which are all ruled by horizontal geodesics. In fact, the vertical plane $y=0$ is also ruled by vertical geodesics, that is, fibers, and this is the only doubly ruled surface among the surfaces in Theorem 2.3. Noting that isometries in $\mathrm{Nil}^{3}$ always move fibers to fibers, we can see that the ruled minimal surfaces in $\mathrm{Nil}^{3}$ always have horizontal ruling geodesics.

Ruled minimal surfaces as limits of helicoids. Consider the (generic) helicoids

$$
H_{\lambda}: y-x \tan (\lambda z)=0
$$

and the point $p_{\lambda}\left(r_{\lambda}, 0,0\right)$ on the $x$-axis, where $r_{\lambda}=\sqrt{2 / \lambda}$. The isometry which sends $x$-axis to itself and sends the origin to $p_{\lambda}$ is given by the left translation by $\left(r_{\lambda}, 0,0\right)$, that is,

$$
(x, y, z) \mapsto\left(r_{\lambda}, 0,0\right) \star(x, y, z)=\left(x+r_{\lambda}, y, z+\frac{r_{\lambda}}{2} y\right) .
$$

If we pull back $H_{\lambda}$ via this isometry, then $p_{\lambda}$ is moved to the origin and the equation of the pullback of $H_{\lambda}$ becomes

$$
y-\left(x+r_{\lambda}\right) \tan \left(\lambda z+\frac{\sqrt{2 \lambda}}{2} y\right)=0 .
$$

Now let $\mu=1 / r_{\lambda}=\sqrt{\lambda / 2}$. Then the above equation can be written as

$$
z=-\frac{y}{2 \mu}+\frac{1}{2 \mu^{2}} \tan ^{-1}\left(\frac{\mu y}{\mu x+1}\right) .
$$


Using the Taylor expansion of $\tan ^{-1}(x)$, we see that this is equivalent to

$$
z=-\frac{x y}{2}+O(\mu)
$$

in a fixed-size box around the origin when $\mu$ is sufficiently small. This function converges uniformly to $z=-x y / 2$ as $\mu$ goes to 0 , which shows that the pointed helicoids $\left(H_{\lambda}, p_{\lambda}\right)$ converge (in the Hausdorff sense) to the exceptional ruled minimal surface $z+x y / 2=0$ :

$$
\left(H_{\lambda}, p_{\lambda}\right) \rightarrow\{z+x y / 2=0\} \quad \text { as } \lambda \rightarrow 0+.
$$

On the other hand, one can easily check that

$$
\begin{array}{ll}
\left(H_{\lambda}, 0\right) \rightarrow \text { horizontal plane } & \text { as } \lambda \rightarrow \infty, \\
\left(H_{\lambda}, 0\right) \rightarrow \text { vertical plane } & \text { as } \lambda \rightarrow 0 .
\end{array}
$$

Therefore all ruled minimal surfaces in $\mathrm{Nil}^{3}$ are either helicoids or limits of sequences of them.

Straight line geodesics. We characterize the geodesics that are straight lines in the Euclidean sense and give another proof of the result in [Bekkar and Sari 1992] mentioned in Section 1.

Proposition 2.4. Let $\gamma(t)=(x(t), y(t), z(t))$ be a geodesic in $\mathrm{Nil}^{3}$.

(1) If $\gamma^{\prime}(0)$ is perpendicular to the fiber, then $\gamma(t)$ is a straight line everywhere perpendicular to the fibers.

(2) If $\gamma^{\prime}(0)$ is parallel to the fiber, then $\gamma(t)$ is a straight line everywhere parallel to the fibers.

Proof. The following equation of geodesics is given in [Inoguchi et al. 1999], but we derive it here again for self-completeness. Note first that

$$
\gamma^{\prime}=x^{\prime} \frac{\partial}{\partial x}+y^{\prime} \frac{\partial}{\partial y}+z^{\prime} \frac{\partial}{\partial z}=x^{\prime} \boldsymbol{e}_{1}+y^{\prime} \boldsymbol{e}_{2}+\left(z^{\prime}+\frac{1}{2}\left(x^{\prime} y-x y^{\prime}\right)\right) \boldsymbol{e}_{3} .
$$

Then we have

$$
\begin{array}{r}
\nabla_{\gamma^{\prime} \gamma^{\prime}=} x^{\prime \prime} \boldsymbol{e}_{1}+y^{\prime \prime} \boldsymbol{e}_{2}+\left(z^{\prime}+\frac{1}{2}\left(x^{\prime} y-x y^{\prime}\right)\right)^{\prime} \boldsymbol{e}_{3} \\
+x^{\prime} \nabla_{\gamma^{\prime}} \boldsymbol{e}_{1}+y^{\prime} \nabla_{\gamma^{\prime}} \boldsymbol{e}_{2}+\left(z^{\prime}+\frac{1}{2}\left(x^{\prime} y-x y^{\prime}\right)\right) \nabla_{\gamma^{\prime}} \boldsymbol{e}_{3} \\
=\left(x^{\prime \prime}+y^{\prime}\left(z^{\prime}+\frac{1}{2}\left(x^{\prime} y-x y^{\prime}\right)\right)\right) \boldsymbol{e}_{1}+\left(y^{\prime \prime}-x^{\prime}\left(z^{\prime}+\frac{1}{2}\left(x^{\prime} y-x y^{\prime}\right)\right)\right) \boldsymbol{e}_{2} \\
+\left(z^{\prime}+\frac{1}{2}\left(x^{\prime} y-x y^{\prime}\right)\right)^{\prime} \boldsymbol{e}_{3} .
\end{array}
$$

Hence $\gamma(t)=(x(t), y(t), z(t))$ is a geodesic if and only if 


$$
\begin{aligned}
x^{\prime \prime}+y^{\prime}\left(z^{\prime}+\frac{1}{2}\left(x^{\prime} y-x y^{\prime}\right)\right) & =0, \\
y^{\prime \prime}-x^{\prime}\left(z^{\prime}+\frac{1}{2}\left(x^{\prime} y-x y^{\prime}\right)\right) & =0, \\
\left(z^{\prime}+\frac{1}{2}\left(x^{\prime} y-x y^{\prime}\right)\right)^{\prime} & =0 .
\end{aligned}
$$

Note that the straight line $(a, b, c t+d)$ parallel to the fiber is a geodesic. Now, suppose $\left\langle\gamma^{\prime}(0), \boldsymbol{e}_{3}\right\rangle=0$. Then, since $\left\langle\gamma^{\prime}(0), \boldsymbol{e}_{3}\right\rangle=\left(z^{\prime}+\frac{1}{2}\left(x^{\prime} y-x y^{\prime}\right)\right)(0)=0$ and since $z^{\prime}+\frac{1}{2}\left(x^{\prime} y-x y^{\prime}\right)$ is a constant function, from the geodesic equation (4) or by the so-called conservation lemma [O'Neill 1983, p. 152], we have $z^{\prime}+\frac{1}{2}\left(x^{\prime} y-x y^{\prime}\right)=0$ for all $t$. Moreover, the geodesic equation (4) gives $x^{\prime \prime}(t)=y^{\prime \prime}(t)=0$, that is, $x(t)$ and $y(t)$ are linear functions of $t$ and consequently from the geodesic equation (4) again, we have

$$
z(t)=-\frac{1}{2}\left(x^{\prime}(0) y(0)-x(0) y^{\prime}(0)\right) t+c
$$

for a constant $c$. Now it is easy to see that $\gamma(t)$ is perpendicular to the fibers everywhere.

If $\gamma^{\prime}(0)$ is parallel to the fiber, then the fiber through $\gamma(0)$ is an image of a geodesic, and from the uniqueness of the geodesic, we have $\gamma(t)=(x(0), y(0), a t+b)$ for constants $a, b$ which is parallel to the fiber everywhere.

Proposition 2.5. Suppose the straight line $\delta(t)=\left(a_{1} t+b_{1}, a_{2} t+b_{2}, a_{3} t+b_{3}\right)$ is a geodesic in $\mathrm{Nil}^{3}$. Then $\delta^{\prime}(0)=\left(a_{1}, a_{2}, a_{3}\right)$ is either perpendicular or parallel to the fiber. Moreover, if $\delta^{\prime}(0)$ is perpendicular to the fiber, then $\delta(t)$ is perpendicular to the fiber everywhere and if $\delta^{\prime}(0)$ is parallel to the fiber, then $\delta(t)$ is parallel to the fiber everywhere.

Proof. In the proof of the above Proposition 2.4, one can see that in order for the straight line $\delta(t)$ to be a geodesic, it should be that $a_{3}=-\frac{1}{2}\left(a_{1} b_{2}-a_{2} b_{1}\right)$. The claims follow easily from this fact.

Now we can also say that every ruled minimal surface in $\mathrm{Nil}^{3}$ is ruled by geodesics which are also straight lines. We remark that it was shown in [Bekkar and Sari 1992] that if the surface is ruled by geodesics that are also straight lines then the surface must be a part of the planes, helicoids or hyperbolic paraboloids. However, in view of Theorem 2.3, we can see that the "straight line" condition is redundant. On the other hand, one may get Theorem 2.3 by applying the aforementioned result together with Lemma 2.1 and Proposition 2.4.

\section{Another characterization of ruled minimal surfaces in $\mathbb{H}_{3}$}

We consider surfaces in $\mathbb{H}_{3}$ whose mean curvature is zero with respect to both metrics $g$ and $g_{L}$ and show that they must be one of (a part of) the above mentioned surfaces, that is, planes, helicoids and hyperbolic paraboloids. 
A Lorentzian connection. Let us consider the left-invariant Lorentzian metric

$$
g_{L}=d x^{2}+d y^{2}-\left(d z+\frac{1}{2}(y d x-x d y)\right)^{2}
$$

on $\mathbb{H}_{3}$ and let $\langle\cdot, \cdot\rangle$ be the Lorentzian inner product. Let $\boldsymbol{e}_{1}, \boldsymbol{e}_{2}$ and $\boldsymbol{e}_{3}$ be the same as the ones given in Section 2. It is easy to show that they are orthonormal with respect to the Lorentzian metric $g_{L}$ as well, that is, $\left\langle\boldsymbol{e}_{i}, \boldsymbol{e}_{j}\right\rangle=0$ if $i \neq j$ and

$$
\left\langle\boldsymbol{e}_{1}, \boldsymbol{e}_{1}\right\rangle=\left\langle\boldsymbol{e}_{2}, \boldsymbol{e}_{2}\right\rangle=1, \quad\left\langle\boldsymbol{e}_{3}, \boldsymbol{e}_{3}\right\rangle=-1 .
$$

Let $D$ be the Levi-Civita connection for the metric $g_{L}$.

Proposition 3.1. We have $D_{\boldsymbol{e}_{i}} \boldsymbol{e}_{i}=0$ for $i=1,2,3$, and

$$
D_{\boldsymbol{e}_{1}} \boldsymbol{e}_{2}=-D_{\boldsymbol{e}_{2}} \boldsymbol{e}_{1}=\frac{1}{2} \boldsymbol{e}_{3}, \quad D_{\boldsymbol{e}_{1}} \boldsymbol{e}_{3}=D_{\boldsymbol{e}_{3}} \boldsymbol{e}_{1}=\frac{1}{2} \boldsymbol{e}_{2}, \quad D_{\boldsymbol{e}_{2}} \boldsymbol{e}_{3}=D_{\boldsymbol{e}_{3}} \boldsymbol{e}_{2}=-\frac{1}{2} \boldsymbol{e}_{1} .
$$

Proof. It is known that the Koszul formula

$2\left\langle\nabla_{V} W, X\right\rangle=V\langle W, X\rangle+W\langle X, V\rangle-X\langle V, W\rangle$

$$
-\langle V,[W, X]\rangle+\langle W,[X, V]\rangle+\langle X,[V, W]\rangle
$$

holds; see, for instance, [O'Neill 1983]. Since $\left[\boldsymbol{e}_{1}, \boldsymbol{e}_{2}\right]=\boldsymbol{e}_{3},\left[\boldsymbol{e}_{2}, \boldsymbol{e}_{3}\right]=\left[\boldsymbol{e}_{3}, \boldsymbol{e}_{1}\right]=0$, one has $\left\langle D_{\boldsymbol{e}_{1}} \boldsymbol{e}_{2}, \boldsymbol{e}_{1}\right\rangle=0,\left\langle D_{\boldsymbol{e}_{1}} \boldsymbol{e}_{2}, \boldsymbol{e}_{2}\right\rangle=0,2\left\langle D_{\boldsymbol{e}_{1}} \boldsymbol{e}_{2}, \boldsymbol{e}_{3}\right\rangle=\left\langle\boldsymbol{e}_{3},\left[\boldsymbol{e}_{1}, \boldsymbol{e}_{2}\right]\right\rangle=\left\langle\boldsymbol{e}_{3}, \boldsymbol{e}_{3}\right\rangle=$ -1 and $D_{\boldsymbol{e}_{1}} \boldsymbol{e}_{2}=\frac{1}{2} \boldsymbol{e}_{3}$. Since

$\left\langle D_{\boldsymbol{e}_{1}} \boldsymbol{e}_{3}, \boldsymbol{e}_{1}\right\rangle=0, \quad\left\langle D_{\boldsymbol{e}_{1}} \boldsymbol{e}_{3}, \boldsymbol{e}_{3}\right\rangle=0, \quad 2\left\langle D_{\boldsymbol{e}_{1}} \boldsymbol{e}_{3}, \boldsymbol{e}_{2}\right\rangle=\left\langle\boldsymbol{e}_{3},\left[\boldsymbol{e}_{2}, \boldsymbol{e}_{1}\right]\right\rangle=\left\langle\boldsymbol{e}_{3},-\boldsymbol{e}_{3}\right\rangle=1$, one has $D_{\boldsymbol{e}_{1}} \boldsymbol{e}_{3}=\frac{1}{2} \boldsymbol{e}_{2}$. One can check the others in the same manner.

Lorentzian exterior product. For tangent vectors

$$
\boldsymbol{v}=a_{1} \boldsymbol{e}_{1}+a_{2} \boldsymbol{e}_{2}+a_{3} \boldsymbol{e}_{3}, \quad \boldsymbol{w}=b_{1} \boldsymbol{e}_{1}+b_{2} \boldsymbol{e}_{2}+b_{3} \boldsymbol{e}_{3}
$$

in $\mathrm{Nil}_{1}^{3}$, the Lorentzian exterior product $\boldsymbol{v} \times_{L} \boldsymbol{w}$ is computed as

$$
\boldsymbol{v} \times_{L} \boldsymbol{w}=\left|\begin{array}{ccc}
\boldsymbol{e}_{1} & \boldsymbol{e}_{2} & -\boldsymbol{e}_{3} \\
a_{1} & a_{2} & a_{3} \\
b_{1} & b_{2} & b_{3}
\end{array}\right|=\left(a_{2} b_{3}-a_{3} b_{2}\right) \boldsymbol{e}_{1}+\left(a_{3} b_{1}-a_{1} b_{3}\right) \boldsymbol{e}_{2}+\left(a_{2} b_{1}-a_{1} b_{2}\right) \boldsymbol{e}_{3},
$$

which is orthogonal to both $\boldsymbol{v}$ and $\boldsymbol{w}$. One can easily see that $\boldsymbol{v} \times_{L} \boldsymbol{w}=\mathbf{0}$ if and only if $\boldsymbol{v}$ and $\boldsymbol{w}$ are linearly dependent.

Zero mean curvature equation. Let $\Sigma$ be a graph of a function $z=f(x, y)$ in $\mathbb{H}_{3}$ and consider the parametrization $\boldsymbol{r}(x, y)=(x, y, f(x, y))$ of $\Sigma$. Set

$$
p=f_{x}+\frac{y}{2}, \quad q=f_{y}-\frac{x}{2} .
$$


If $\Sigma$ is minimal, that is, the mean curvature is zero in $\mathrm{Nil}^{3}$, the function $f$ satisfies the minimal surface equation

$$
\left(1+q^{2}\right) f_{x x}-2 p q f_{x y}+\left(1+p^{2}\right) f_{y y}=0 .
$$

For the derivation of this equation, see for example [Inoguchi et al. 2000].

In this section, we will derive an equation for the mean curvature of the graph $\Sigma$ to be zero with respect to the Lorentzian metric $g_{L}$. First, let us recall some definitions. A point $z \in \Sigma$ is called spacelike if the induced metric on $T_{z} \Sigma$ is Riemannian, timelike if the induced metric is Lorentzian and lightlike if the induced metric has rank 1 . We will derive the equation when $\Sigma$ is spacelike, that is, every point of $\Sigma$ is a spacelike point. The case when $\Sigma$ is timelike is almost identical. Note that when $z \in \Sigma$ is lightlike, one cannot define the mean curvature.

Now let $\Sigma$ be a spacelike graph of a function $z=f(x, y)$. Note first that $p^{2}+q^{2}<1$ since the graph is spacelike. We now compute the first fundamental form I and the second fundamental form II of $\Sigma$. Since

$$
\boldsymbol{r}_{x}=\left(1,0, f_{x}\right)=\boldsymbol{e}_{1}+p \boldsymbol{e}_{3}, \quad \boldsymbol{r}_{y}=\left(0,1, f_{y}\right)=\boldsymbol{e}_{2}+q \boldsymbol{e}_{3}
$$

and

$$
\begin{aligned}
& \left\langle\boldsymbol{r}_{x}, \boldsymbol{r}_{x}\right\rangle=1-p^{2}, \\
& \left\langle\boldsymbol{r}_{x}, \boldsymbol{r}_{y}\right\rangle=-p q, \\
& \left\langle\boldsymbol{r}_{y}, \boldsymbol{r}_{y}\right\rangle=1-q^{2},
\end{aligned}
$$

one has

$$
\begin{aligned}
& E=\left\langle\boldsymbol{r}_{x}, \boldsymbol{r}_{x}\right\rangle=1-p^{2}, \\
& F=\left\langle\boldsymbol{r}_{x}, \boldsymbol{r}_{y}\right\rangle=-p q, \\
& G=\left\langle\boldsymbol{r}_{y}, \boldsymbol{r}_{y}\right\rangle=1-q^{2} .
\end{aligned}
$$

Since $\boldsymbol{r}_{x} \times_{L} \boldsymbol{r}_{y}=-p \boldsymbol{e}_{1}-q \boldsymbol{e}_{2}-\boldsymbol{e}_{3}$, the unit normal vector field $\boldsymbol{n}$ to the graph is

$$
\boldsymbol{n}=\frac{1}{W}\left(-p \boldsymbol{e}_{1}-q \boldsymbol{e}_{2}-\boldsymbol{e}_{3}\right), \quad W=\sqrt{1-\left(p^{2}+q^{2}\right)} .
$$

Since the directional derivatives of $p$ and $q, \boldsymbol{e}_{i}(p)$ and $\boldsymbol{e}_{i}(q)$, are computed as

$$
\begin{aligned}
& \boldsymbol{e}_{1}(p)=\left(\frac{\partial}{\partial x}-\frac{y}{2} \frac{\partial}{\partial z}\right)\left(f_{x}+\frac{y}{2}\right)=f_{x x}, \\
& \boldsymbol{e}_{1}(q)=\left(\frac{\partial}{\partial x}-\frac{y}{2} \frac{\partial}{\partial z}\right)\left(f_{y}-\frac{x}{2}\right)=f_{x y}-\frac{1}{2}, \\
& \boldsymbol{e}_{2}(p)=\left(\frac{\partial}{\partial y}+\frac{x}{2} \frac{\partial}{\partial z}\right)\left(f_{x}+\frac{y}{2}\right)=f_{x y}+\frac{1}{2}, \\
& \boldsymbol{e}_{2}(q)=\left(\frac{\partial}{\partial y}+\frac{x}{2} \frac{\partial}{\partial z}\right)\left(f_{y}-\frac{x}{2}\right)=f_{y y},
\end{aligned}
$$


one has

$$
\begin{aligned}
& D_{\boldsymbol{r}_{x}} \boldsymbol{r}_{x}=D_{\left(\boldsymbol{e}_{1}+p \boldsymbol{e}_{3}\right)}\left(\boldsymbol{e}_{1}+p \boldsymbol{e}_{3}\right)=p \boldsymbol{e}_{2}+f_{x x} \boldsymbol{e}_{3}, \\
& D_{\boldsymbol{r}_{y}} \boldsymbol{r}_{x}=-\frac{p}{2} \boldsymbol{e}_{1}+\frac{q}{2} \boldsymbol{e}_{2}+f_{x y} \boldsymbol{e}_{3}, \\
& D_{\boldsymbol{r}_{y}} \boldsymbol{r}_{y}=-q \boldsymbol{e}_{1}+f_{y y} \boldsymbol{e}_{3} .
\end{aligned}
$$

Then one has the following coefficients of the second fundamental form II:

$$
\begin{aligned}
l & =\left\langle D_{\boldsymbol{r}_{x}} \boldsymbol{r}_{x}, \boldsymbol{n}\right\rangle=\frac{1}{W}\left(-p q+f_{x x}\right), \\
m & =\left\langle D_{\boldsymbol{r}_{y}} \boldsymbol{r}_{x}, \boldsymbol{n}\right\rangle=\frac{1}{W}\left(\frac{p^{2}}{2}-\frac{q^{2}}{2}+f_{x y}\right), \\
n & =\left\langle D_{\boldsymbol{r}_{y}} \boldsymbol{r}_{y}, \boldsymbol{n}\right\rangle=\frac{1}{W}\left(p q+f_{y y}\right) .
\end{aligned}
$$

Now the mean curvature $H$ of the spacelike graph $\Sigma$ is computed as

$$
H=\frac{1}{2} \frac{l G-2 m F+n E}{E G-F^{2}} .
$$

Then, since

$$
\begin{array}{rl}
l G-2 & m F+n E \\
= & \frac{1}{W}\left[\left(-p q+f_{x x}\right)\left(1-q^{2}\right)+\left(\frac{p^{2}-q^{2}}{2}+f_{x y}\right) p q+\left(p q+f_{y y}\right)\left(1-p^{2}\right)\right] \\
= & \frac{1}{W}\left[\left(1-q^{2}\right) f_{x x}+2 p q f_{x y}+\left(1-p^{2}\right) f_{y y}\right],
\end{array}
$$

one can see that the mean curvature of the graph $z=f(x, y)$ of a function $f(x, y)$ is zero if and only if

$$
\left(1-q^{2}\right) f_{x x}+2 p q f_{x y}+\left(1-p^{2}\right) f_{y y}=0 .
$$

When the graph $\Sigma$ is timelike, one has the same equation.

\section{Zero mean curvature surface.}

Theorem 3.2. Let $\Sigma$ be a surface in $\mathbb{H}_{3}$. If the mean curvature of $\Sigma$ is zero with respect to both metrics $g$ and $g_{L}$, then up to the isometries in $\mathrm{Nil}^{3}, \Sigma$ is contained in one of these surfaces:

- the horizontal plane $z=0$;

- the vertical plane $y=0$;

- a helicoid $\tan (\lambda z)=y / x, \lambda \neq 0$;

- a hyperbolic paraboloid $z=-x y / 2$. 
Proof. Suppose first that $\Sigma$ has a point around which it can be represented as a graph of a function of $(x, y)$, say, $z=f(x, y)$. Consider the vector field $X=-q \boldsymbol{e}_{1}+p \boldsymbol{e}_{2}$. Since $X=-q \boldsymbol{e}_{1}+p \boldsymbol{e}_{2}=-q \boldsymbol{r}_{x}+p \boldsymbol{r}_{y}$, it is tangent to $\Sigma$. Since the vector $N=\boldsymbol{r}_{x} \times \boldsymbol{r}_{y}=-p \boldsymbol{e}_{1}-q \boldsymbol{e}_{2}-\boldsymbol{e}_{3}$ is orthogonal to $\Sigma$ and since $N \times \boldsymbol{e}_{3}=-q \boldsymbol{e}_{1}+p \boldsymbol{e}_{2}=X, X$ is orthogonal to both $N$ and $\boldsymbol{e}_{3}$. Then one has

$$
\nabla_{X} X=\left(q\left(f_{x y}-\frac{1}{2}\right)-p f_{y y}\right) \boldsymbol{e}_{1}+\left(p\left(f_{x y}+\frac{1}{2}\right)-q f_{x x}\right) \boldsymbol{e}_{2} .
$$

Now, since the mean curvature of $\Sigma \subset \mathbb{H}_{3}$ is zero with respect to both $g$ and $g_{L}$, one has

$$
\begin{aligned}
& \left(1+q^{2}\right) f_{x x}-2 p q f_{x y}+\left(1+p^{2}\right) f_{y y}=0, \\
& \left(1-q^{2}\right) f_{x x}+2 p q f_{x y}+\left(1-p^{2}\right) f_{y y}=0 .
\end{aligned}
$$

Subtracting the two equations, one has

$$
q^{2} f_{x x}-2 p q f_{x y}+p^{2} f_{y y}=0
$$

and then one has finally by (7),

$$
\begin{aligned}
X \times \nabla_{X} X & =\left(-q \boldsymbol{e}_{1}+p \boldsymbol{e}_{2}\right) \times\left[\left(q\left(f_{x y}-\frac{1}{2}\right)-p f_{y y}\right) \boldsymbol{e}_{1}+\left(p\left(f_{x y}+\frac{1}{2}\right)-q f_{x x}\right) \boldsymbol{e}_{2}\right] \\
& =\left(q^{2} f_{x x}-2 p q f_{x y}+p^{2} f_{y y}\right) \boldsymbol{e}_{3}=0 .
\end{aligned}
$$

Now, since $X$ and $\nabla_{X} X$ have the same direction, the integral curve of $X$ passing through a point in $\Sigma$ is a geodesic, and since $X$ is orthogonal to $\boldsymbol{e}_{3}$, the geodesic is orthogonal to the fiber. Hence the surface $\Sigma$ is a horizontally ruled minimal surface in $\mathrm{Nil}^{3}$.

If the surface $\Sigma$ has no point around which $\Sigma$ is represented as the graph of $f(x, y)$, then it is a vertical cylinder over a curve in the $x y$-plane and has a parametrization

$$
X(s, t)=(x(s), y(s), t),
$$

with $x(0)=y(0)=0$. By repeating the arguments in Theorem 2.3, one can show that the surface is isometric to the vertical plane $y=0$. Now this completes the proof.

Remark. If we add (5) and (6), we have $f_{x x}+f_{y y}=0$, that is, if a graph of a function $z=f(x, y)$ in $\mathbb{H}_{3}$ satisfies the condition of Theorem 3.2, $f$ must be a harmonic function. This fact is true for the three-dimensional Lorentzian space $\mathbb{L}^{3}$ and is the motivation of [Kim et al. 2009b]. We think it is a nontrivial fact and would like to find applications of this fact in future study. 


\section{Acknowledgments}

We would like to express our gratitude to the anonymous referee for many valuable suggestions.

\section{References}

[Albujer and Alías 2009] A. L. Albujer and L. J. Alías, "Calabi-Bernstein results for maximal surfaces in Lorentzian product spaces”, J. Geom. Phys. 59:5 (2009), 620-631. MR 2010i:47088 Zbl 1173.53025

[Alías and Palmer 2001] L. J. Alías and B. Palmer, "A duality result between the minimal surface equation and the maximal surface equation", An. Acad. Brasil. Ciênc. 73:2 (2001), 161-164. MR 2002c:53007 Zbl 0999.53007

[Bekkar and Sari 1992] M. Bekkar and T. Sari, "Surfaces minimales réglées dans l'espace de Heisenberg $\mathbf{H}_{3}$ ”, Rend. Sem. Mat. Univ. Politec. Torino 50:3 (1992), 243-254. MR 94h:53009 Zbl 0810.53012

[Bekkar et al. 2007] M. Bekkar, F. Bouziani, Y. Boukhatem, and J. Inoguchi, "Helicoids and axially symmetric minimal surfaces in 3-dimensional homogeneous spaces", Differ. Geom. Dyn. Syst. 9 (2007), 21-39. MR 2008e:53009 Zbl 1159.53335

[Daniel 2011] B. Daniel, "The Gauss map of minimal surfaces in the Heisenberg group", Int. Math. Res. Not. 2011:3 (2011), 674-695. MR 2012b:53117 Zbl 1209.53048

[Inoguchi 2005] J.-i. Inoguchi, "Flat translation invariant surfaces in the 3-dimensional Heisenberg group", J. Geom. 82:1-2 (2005), 83-90. MR 2006d:53070 Zbl 1082.53064

[Inoguchi 2008] J.-i. Inoguchi, "Minimal surfaces in the 3-dimensional Heisenberg group", Differ. Geom. Dyn. Syst. 10 (2008), 163-169. MR 2009a:53009 Zbl 1160.53364

[Inoguchi et al. 1999] J.-i. Inoguchi, T. Kumamoto, N. Ohsugi, and Y. Suyama, "Differential geometry of curves and surfaces in 3-dimensional homogeneous spaces, I", Fukuoka Univ. Sci. Rep. 29:2 (1999), 155-182. MR 2000h:53018 Zbl 0962.53032

[Inoguchi et al. 2000] J.-I. Inoguchi, T. Kumamoto, N. Ohsugi, and Y. Suyama, "Differential geometry of curves and surfaces in 3-dimensional homogeneous spaces, II", Fukuoka Univ. Sci. Rep. 30:1 (2000), 17-47. MR 2001c:53085 Zbl 0974.53039

[Kim et al. 2009a] Y. W. Kim, S.-E. Koh, H. Shin, and S.-D. Yang, "Helicoids in $\mathbb{S}^{2} \times \mathbb{R}$ and $\mathbb{H}^{2} \times \mathbb{R}$ ", Pacific J. Math. 242:2 (2009), 281-297. MR 2010h:53008 Zbl 1172.53037

[Kim et al. 2009b] Y. W. Kim, H. Y. Lee, and S.-D. Yang, "Minimal harmonic graphs and their Lorentzian cousins”, J. Math. Anal. Appl. 353:2 (2009), 666-670. MR 2010i:53110 Zbl 1161.53051

[Kobayashi 1983] O. Kobayashi, "Maximal surfaces in the 3-dimensional Minkowski space $L^{3}$ ", Tokyo J. Math. 6:2 (1983), 297-309. MR 85d:53003 Zbl 0535.53052

[Lee 2011] H. Lee, "Maximal surfaces in Lorentzian Heisenberg space", Differential Geom. Appl. 29:1 (2011), 73-84. MR 2012e:53117 Zbl 1216.53055

[Mercuri et al. 2006] F. Mercuri, S. Montaldo, and P. Piu, "A Weierstrass representation formula

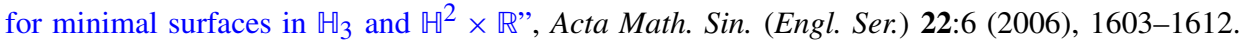
MR 2007g:53007 Zbl 1119.53041

[O’Neill 1983] B. O’Neill, Semi-Riemannian geometry: with applications to relativity, Pure and Applied Mathematics 103, Academic Press, New York, 1983. MR 85f:53002 Zbl 0531.53051

[Rahmani 1992] S. Rahmani, "Métriques de Lorentz sur les groupes de Lie unimodulaires, de dimension trois", J. Geom. Phys. 9:3 (1992), 295-302. MR 93f:53061 Zbl 0752.53036 
[Rahmani and Rahmani 2006] N. Rahmani and S. Rahmani, "Lorentzian geometry of the Heisenberg group”, Geom. Dedicata 118 (2006), 133-140. MR 2007h:53112 Zbl 1094.53065

[Sanini 1997] A. Sanini, "Gauss map of a surface of the Heisenberg group", Boll. Un. Mat. Ital. B (7) 11:2, suppl. (1997), 79-93. MR 98e:53009 Zbl 0886.53018

Received October 6, 2011. Revised September 14, 2012.

\section{HEAYONG SHIN}

DEPARTMENT OF MATHEMATICS

CHUNG-ANG UNIVERSITY

SEOUL 156-756

SOUTH KOREA

hshin@cau.ac.kr

Young WoOK KIM

DEPARTMENT OF MATHEMATICS

KOREA UNIVERSITY

SEOUL 136-701

SOUTH KOREA

ywkim@korea.ac.kr

Sung-Eun KOH

DEPARTMENT OF MATHEMATICS

KONKUK UNIVERSITY

SEOUL 143-701

SOUTH KOREA

sekoh@konkuk.ac.kr

HYUNG Yong LEE

DEPARTMENT OF MATHEMATICS

KOREA UNIVERSITY

SEOUL 136-713

SOUTH KoreA

distgeo@korea.ac.kr

SEONG-DEOG YANG

DEPARTMENT OF MATHEMATICS

KOREA UNIVERSITY

SEOUL 136-713

SOUTH KOREA

sdyang@korea.ac.kr 


\title{
PACIFIC JOURNAL OF MATHEMATICS
}

\author{
msp.org/pjm
}

Founded in 1951 by E. F. Beckenbach (1906-1982) and F. Wolf (1904-1989)

\section{EDITORS}

V. S. Varadarajan (Managing Editor)

Department of Mathematics

University of California

Los Angeles, CA 90095-1555

pacific@math.ucla.edu

Paul Balmer

Department of Mathematics

University of California

Los Angeles, CA 90095-1555

balmer@math.ucla.edu

Daryl Cooper

Department of Mathematics

University of California

Santa Barbara, CA 93106-3080 cooper@math.ucsb.edu

Jiang-Hua $\mathrm{Lu}$

Department of Mathematics

The University of Hong Kong

Pokfulam Rd., Hong Kong jhlu@maths.hku.hk
Don Blasius

Department of Mathematics University of California

Los Angeles, CA 90095-1555

blasius@math.ucla.edu

Robert Finn

Department of Mathematics Stanford University

Stanford, CA 94305-2125

finn@math.stanford.edu

Sorin Popa

Department of Mathematics

University of California

Los Angeles, CA 90095-1555

popa@math.ucla.edu

Paul Yang

Department of Mathematics

Princeton University

Princeton NJ 08544-1000

yang@math.princeton.edu

\section{PRODUCTION}

Silvio Levy, Scientific Editor, production@msp.org

\section{SUPPORTING INSTITUTIONS}

ACADEMIA SINICA, TAIPEI

CALIFORNIA INST. OF TECHNOLOGY

INST. DE MATEMÁTICA PURA E APLICADA

KEIO UNIVERSITY

MATH. SCIENCES RESEARCH INSTITUTE

NEW MEXICO STATE UNIV.

OREGON STATE UNIV.

\author{
STANFORD UNIVERSITY \\ UNIV. OF BRITISH COLUMBIA \\ UNIV. OF CALIFORNIA, BERKELEY \\ UNIV. OF CALIFORNIA, DAVIS \\ UNIV. OF CALIFORNIA, LOS ANGELES \\ UNIV. OF CALIFORNIA, RIVERSIDE \\ UNIV. OF CALIFORNIA, SAN DIEGO \\ UNIV. OF CALIF., SANTA BARBARA
}

\author{
Vyjayanthi Chari \\ Department of Mathematics \\ University of California \\ Riverside, CA 92521-0135 \\ chari@math.ucr.edu \\ Kefeng Liu \\ Department of Mathematics \\ University of California \\ Los Angeles, CA 90095-1555 \\ liu@math.ucla.edu \\ Jie Qing \\ Department of Mathematics \\ University of California \\ Santa Cruz, CA 95064 \\ qing@cats.ucsc.edu
}

These supporting institutions contribute to the cost of publication of this Journal, but they are not owners or publishers and have no responsibility for its contents or policies.

See inside back cover or msp.org/pjm for submission instructions.

The subscription price for 2013 is US \$400/year for the electronic version, and \$485/year for print and electronic.

Subscriptions, requests for back issues and changes of subscribers address should be sent to Pacific Journal of Mathematics, P.O. Box 4163, Berkeley, CA 94704-0163, U.S.A. The Pacific Journal of Mathematics is indexed by Mathematical Reviews, Zentralblatt MATH, PASCAL CNRS Index, Referativnyi Zhurnal, Current Mathematical Publications and the Science Citation Index.

The Pacific Journal of Mathematics (ISSN 0030-8730) at the University of California, c/o Department of Mathematics, 798 Evans Hall \#3840, Berkeley, CA 94720-3840, is published monthly except July and August. Periodical rate postage paid at Berkeley, CA 94704, and additional mailing offices. POSTMASTER: send address changes to Pacific Journal of Mathematics, P.O. Box 4163, Berkeley, CA 94704-0163.

PJM peer review and production are managed by EditFLOW ${ }^{\circledR}$ from Mathematical Sciences Publishers.

PUBLISHED BY

mathematical sciences publishers

nonprofit scientific publishing

http://msp.org/

(C) 2013 Mathematical Sciences Publishers 


\section{PACIFIC JOURNAL OF MATHEMATICS}

Volume $261 \quad$ No. $2 \quad$ February 2013

Geography of simply connected nonspin symplectic 4-manifolds with positive signature

Anar Akhmedov, Mark C. Hughes and B. Doug Park

Schur-Horn theorems in $\mathrm{II}_{\infty}$-factors

283

MARTÍN ARGERAMI and PEDRo MASSEY

Classification of positive solutions for an elliptic system with a higher-order

fractional Laplacian

JiNGBo DOU and CHANGZHENG QU

Bound states of asymptotically linear Schrödinger equations with compactly supported potentials

MingWEN FEI and HUICHENG YiN

Type I almost homogeneous manifolds of cohomogeneity one, III

DANIEL GUAN

The subrepresentation theorem for automorphic representations

MARCELA HANZER

Variational characterizations of the total scalar curvature and eigenvalues of the

Laplacian

Seungsu Hwang, JeOngwook Chang and GabJin Yun

Fill-ins of nonnegative scalar curvature, static metrics, and quasi-local mass

JEFFREY L. JAUREGUI

Operator algebras and conjugacy problem for the pseudo-Anosov automorphisms of a surface

\section{IGOR NIKOLAEV}

Connected sums of closed Riemannian manifolds and fourth-order conformal invariants

DAVID RASKE

Ruled minimal surfaces in the three-dimensional Heisenberg group

Heayong Shin, Young Wook Kim, Sung-Eun Koh, Hyung Yong LeE and SEONG-DEOG YANG

$G$-bundles over elliptic curves for non-simply laced Lie groups and configurations of lines in rational surfaces

MANG XU and JIAJIN ZHANG 